Report No.: ORNL/TM-2000/265

\title{
NERI PROJECT 99-119. "A NEW PARADIGM FOR AUTOMATIC DEVELOPMENT OF HIGHLY RELIABLE CONTROL ARCHITECTURES FOR NUCLEAR POWER PLANTS." PHASE-1 PROGRESS REPORT
}

J. March-Leuba

R. T. Wood

Oak Ridge National Laboratory

B. R. Upadyahya

The University of Tennessee, Knoxville

J. M. Doster

C. W. Mayo

North Carolina State University

August 2000

Prepared by the

Oak Ridge National Laboratory

Oak Ridge, Tennessee 37831

Managed by

UT-Battelle, LLC

For

U.S. Department of Energy

Under contract DE-AC05-00OR22725 


\section{DISCLAIMER}

This report was prepared as an account of work sponsored by an agency of the United States government. Neither the United States Government nor any agency thereof, nor any of their employees, makes any warranty, express or implied, or assumes any legal liability or responsibility for the accuracy, completeness, or usefulness of any information, apparatus, product, or process disclosed, or represents that its use would not infringe privately owned rights. Reference herein to any specific commercial product, process, or service by trade name, trademark, manufacturer, or otherwise, does not necessarily constitute or imply its endorsement, recommendation, or favoring by the United States Government or any agency thereof. The views and opinions of authors expressed herein do not necessarily state or reflect those of the United States Government or any agency thereof. 


\section{TABLE OF CONTENTS}

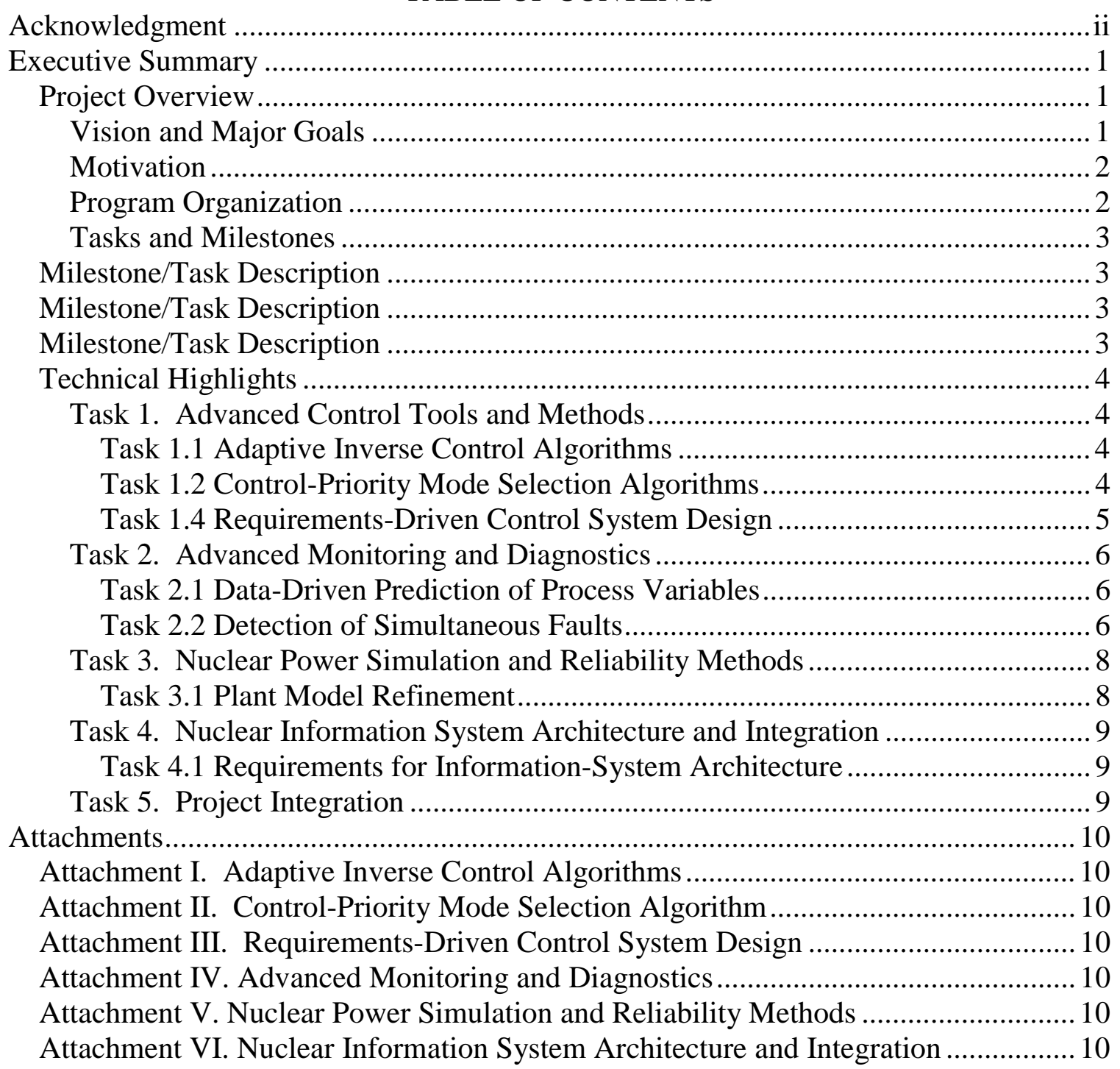




\section{Acknowledgment}

This research has been conducted under a grant funded by the U.S. Department of Energy Nuclear Energy Research Initiative (NERI,) project number NERI 99-119. The first page of this report lists as authors the principal investigators from the three organizations. A detailed list of all major contributors follows

C. R. Brittain (ORNL)

N. E. Clapp (ORNL)

W. Dong (NCSU)

J. M. Doster (NCSU)

M. Fisher (NCSU)

I. Goncalves (UTK)

N. Kaista (UTK)

H. E. Knee (ORNL)

B. Lu (UTK)

J. March-Leuba (ORNL)

J. A. Mullens (ORNL)

C. W. Mayo (NCSU)

A. Sakabe (NCSU)

A. A. Shourbaji (ORNL)

B. R. Upadyahya (UTK)

R. T. Wood (ORNL) 


\section{Executive Summary}

This report describes the tasks performed and the progress made during Phase 1 of the DOE-NERI project number 99-119 entitled Automatic Development of Highly Reliable Control Architecture for Future Nuclear Power Plants. This project is a collaboration effort between the Oak Ridge National Laboratory (ORNL,) The University of Tennessee, Knoxville (UTK) and the North Carolina State University (NCSU). ORNL is the lead organization and is responsible for the coordination and integration of all work.

\section{Project Overview}

\section{Vision and Major Goals}

This research focuses on the development of methods for automated generation of control systems that can be traced directly to the design requirements for the life of the plant.

Our final goal is to "capture" the design requirements inside a "control engine" during the design phase. This control engine is, then, not only capable of designing automatically the initial implementation of the control system, but it also can confirm that the original design requirements are still met during the life of the plant as conditions change.

This control engine captures the high-level requirements and stress factors that the control system must survive (e.g. a list of transients, or a requirement to withstand a single failure.) The control engine, then, is able to generate automatically the controlsystem algorithms and parameters that optimize a design goal and satisfy all requirements. As conditions change during the life of the plant (e.g. component degradation, or subsystem failures) the control engine automatically "flags" that a requirement is not satisfied, and it can even suggest a modified configuration that would

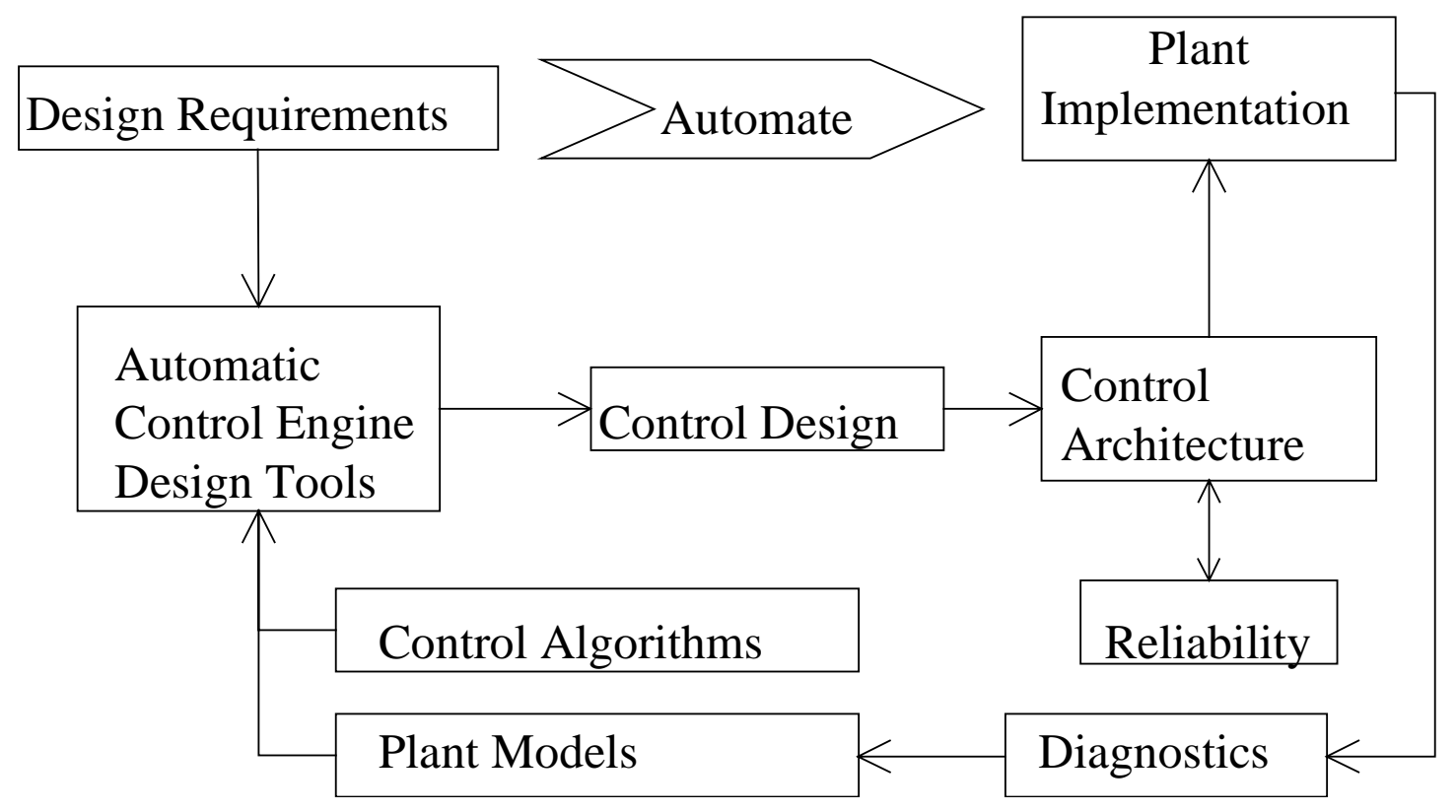

Figure 1. Schematic diagram of the automated control design process 
satisfy it. This control engine concept is shown schematically in Fig. 1.

The implementation of this "control-engine" design methodology requires the following steps, which are described in detail in the attachments to this report:

1. Selection of Design Requirements Related to Control System Performance

2. Implementation of Requirements in Mathematical Form

3. Development of a Control Algorithm Library

4. Development and Validation of Plant Models

5. Automated Control Design Development

6. Development of Control Architectures

7. Control Design Implementation

8. Development of Diagnostics Methods to Update the Plant Model

\section{Motivation}

In the past two decades there have been significant progress in the automated development of control algorithms and on computer aids for fast and reliable implementation of those algorithms. Indeed, there are a number of commercial off-theshelf software packages that can help design and parameterize control systems. Some of these packages even have graphical interfaces, which facilitate the use of alreadyvalidated software modules. It is not the goal of this research to reproduce this existing work

The purpose of this research is to extend the current state of the art, so that the design requirements are captured in a control engine, which is used during the life of the facility to confirm that the original requirements are still met as plant conditions change.

Capturing these requirements is of special relevance to the nuclear industry, where the plant life is often 40 to 60 years, and the system requirements are not always obvious.

Nuclear plant availability has improved significantly in the past decade. One reason for this improvement is that plants are relying more heavily on the control system response to prevent unnecessary scrams. Thus, improved economic operation of the plant relies more and more on these "hidden" requirements, where the control system is quite capable of maintaining steady state or normal operation, but cannot handle a postulated transient because of a degraded condition. By capturing the requirements into the control system itself, we can make the operator aware of this deficiency, so that either the degraded condition can be corrected in time, or the control system updated to handle it.

\section{Program Organization}

This project is a collaborative effort between the Oak Ridge National Laboratory (ORNL), The University of Tennessee, Knoxville (UTK), and the North Carolina State University (NCSU). ORNL is the lead organization and is responsible for the coordination and integration of all work. The program is organized into five major tasks, which address the major steps described above.

1. Advanced Control Tools and Methods. ORNL is the responsible organization. 
2. Advanced Monitoring and Diagnostics. UTK is the responsible organization.

3. Nuclear Power Simulation and Reliability Methods. NCSU is the responsible organization.

4. Nuclear Information System Architecture and Integration. ORNL is the responsible organization.

5. Project Integration. ORNL is the responsible organization.

\section{Tasks and Milestones}

All tasks and milestones for Phase 1 have been completed and detailed reports on each of them are attached to this summary. The following tables shows a listing of all tasks for each of the three project phases along with the responsible organization and planned completion dates.

\section{Phase 1:}

\begin{tabular}{|l|l|l|l|}
\hline \multirow{2}{*}{ Milestone/Task Description } & \multirow{2}{*}{ Org. } & \multicolumn{2}{|c|}{ Completion Date } \\
\cline { 3 - 4 } & & Planned & Actual \\
\hline 1.1 Adaptive Inverse Control Algorithms & ORNL & $6 / 00$ & $6 / 00$ \\
\hline 1.2 Control-Priority Mode Selection Algorithms & ORNL & $9 / 00$ & $7 / 00$ \\
\hline 2.1 Data-Driven Prediction of Process Variables & UTK & $6 / 00$ & $6 / 00$ \\
\hline 2.2 Detection of Simultaneous Faults & UTK & $9 / 00$ & $7 / 00$ \\
\hline 3.1 Plant Model Refinement & NCSU & $9 / 00$ & $8 / 00$ \\
\hline 4.1 Requirements for Information-System Architecture & ORNL & $9 / 00$ & $7 / 00$ \\
\hline 5.1 Development of Integrated Software & ORNL & $9 / 00$ & $7 / 00$ \\
\hline
\end{tabular}

\section{Phase 2:}

\begin{tabular}{|l|l|l|l|} 
Milestone/Task Description & \multirow{2}{*}{ Org. } & \multicolumn{2}{|c|}{ Completion Date } \\
\cline { 3 - 4 } 1.3 Controllers for Feedwater Systems & & Planned & Actual \\
\hline 1.4 Requirements-Driven Control System Design & ORNL & $9 / 01$ & \\
\hline 2.3 Implementation of On-line Diagnostics System & ORNL & $6 / 02$ & \\
\hline 3.2 Reliability Analysis Methods Development & NCSU & $9 / 01$ & \\
\hline 3.3 Reliability Analysis Methods Integration & NCSU & $6 / 01$ & \\
\hline 4.2 Application Programming Interfaces & ORNL & $6 / 01$ & \\
\hline 4.3 Application Environment & ORNL & $9 / 01$ & \\
\hline 5.1 Development of Integrated Software & ORNL & $9 / 01$ & \\
\hline
\end{tabular}

\section{Phase 3:}

\begin{tabular}{|l|l|l|l|}
\hline \multirow{2}{*}{ Milestone/Task Description } & \multirow{2}{*}{ Org. } & \multicolumn{2}{|c|}{ Completion Date } \\
\cline { 3 - 4 } & & Planned & Actual \\
\hline 1.4 Requirements-Driven Control System Design & ORNL & $6 / 02$ & \\
\hline 2.4 Requirements for Implementation on FDI System & UTK & $9 / 02$ & \\
\hline 3.3 Reliability Analysis Methods Integration & NCSU & $6 / 02$ & \\
\hline 4.4 Integrated Applications & ORNL & $9 / 02$ & \\
\hline 5.1 Development of Integrated Software & ORNL & $9 / 02$ & \\
\hline
\end{tabular}




\section{Technical Highlights}

\section{Task 1. Advanced Control Tools and Methods}

The ultimate goal of this task is to develop and demonstrate the control-engine concept. To this end, we have developed libraries of control algorithms, and we have successfully applied some of them to a prototype control engine problem.

\section{Task 1.1 Adaptive Inverse Control Algorithms}

Under this task, we have developed a general methodology for handling the sensor and actuator nonlinearities as piecewise linear functions. The piecewise linear functions provide very good approximations to characteristics such as hysteresis, dead zone, and backlash. The piecewise functions have sufficient number of free parameters to permit a realistic representation of most physical situations that occur with valves, rod controls, and other types of devices that occur in power plant control loops. The adaptative iteration updates the parameter estimates in the inverse law. Since such parameters are usually not known and can change with component wear and operating conditions, the advantage of the adaptive inverse control strategy is that it can adjust automatically using operating data. This type of algorithm eliminates the need for information from higher levels of the control algorithm to handle the performance characteristics. We have implemented inverse control algorithms for different non-linear actuator problems, such as dead-zone compensation or backslash non-linearity. The details are shown in Attachment I.

Figures 2 through 4 show an example application of this technique to an actuator with a dead zone. As seen in Figure 4, the inverse control algorithm removes completely the non-linearity and allows for a simpler and more robust control system design.

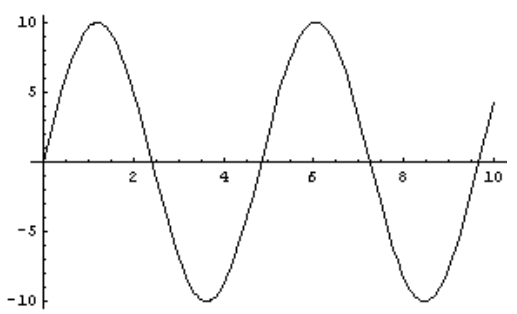

Figure 2. Input signal to actuator.

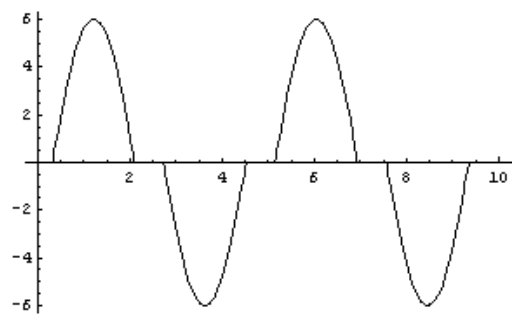

Figure 3. Output signal from nonlinear actuator with dead zone.

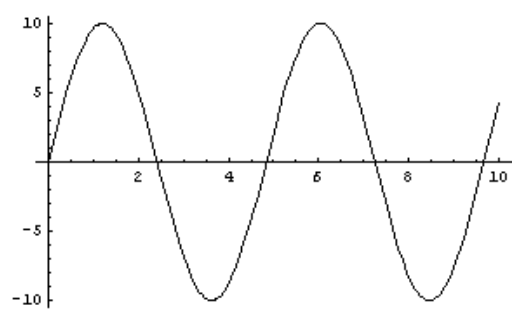

Figure 4. Output signal from nonlinear actuator with dead zone compensation.

\section{Task 1.2 Control-Priority Mode Selection Algorithms}

Control priority mode selection is a strategy that allows autonomous agents to select the best control mode for a given condition. The control system designer provides detailed information needed to select the highest priority regulation objectives required to maintain the controllability of the full system for different failure states of actuators. For example, when one actuator saturates or is placed in manual, that actuator no longer responds to automatic control signals. The controllability of the full system is reduced in rank by one degree due to the loss of one actuator. The highest regulation objective is 
changed to maintain the most important objective in response to a reduced controllability rank of the full system.

Control priority mode selection has been implemented using the "socket" algorithms described in Attachment II. These control sockets use information from several sources to select the optimal algorithm given the current circumstances based on the entropy value calculated for each option. These sources of information include the on-line diagnostic systems, a library of available control algorithms, and repair and maintenance information. Proof of principle of this algorithm has been successfully demonstrated using a nine-component system with two possible control algorithms.

\section{Task 1.4 Requirements-Driven Control System Design}

Work under this task has concentrated on developing methods for automated generation of control systems that can be traced directly to the design requirements. Our final goal is to allow the control designer to specify only high-level requirements and stress factors that the control system must accomodate (e.g. a list of transients, or a requirement to withstand a single failure.) To this end, the "control engine" automatically selects and validates control algorithms and parameters that are optimized to the current state of the plant, and that have been tested under the prescribed stress factors. The control engine then automatically generates the control software from validated algorithms.

The implementation of the methodology takes the bottom path of Fig. 1. For this implementation, the design requirements are fed into a control engine, which uses a library of control algorithms and validated plant models to arrive at the control design using an iterative optimization process. The control design is then implemented using validated control architectures, which are tested automatically to guarantee that the reliability requirements are met. Finally, during the lifetime of the plant, the plant model is kept current (e.g, updated with component failures or mode changes) by an on-line diagnostics system.

As a demonstration of proof of principle, we have implemented this methodology using a standard off-the-shelf minimization algorithm. For this example, we have used a simplified steam generator model (Fig 6) where the maximum flow through the inlet valve is limited by the full-open stem position, which introduces a hard nonlinearity. The details of this research are described in Attachment III. Figure 5 shows an example of the application of the control-engine methodology. For this example, the controller is required to prevent a low/high level scram following a 50\% step in load demand. While, on the surface, this example appears to be almost trivial, the solution is a lot harder than it appears because of the non-linear nature of the problem. In the first few seconds of the transient, the so-called "shrink and swell" phenomena are apparent, where the water level drops even though the steam generation has been reduced. These types of systems cause severe problems for most automated control generation schemes which rely on linearization around the equilibrium point. For example, if we linearize this system around time zero, we observe that a reduction in power requires an increase of inlet flow, while at longer times the opposite is required. 


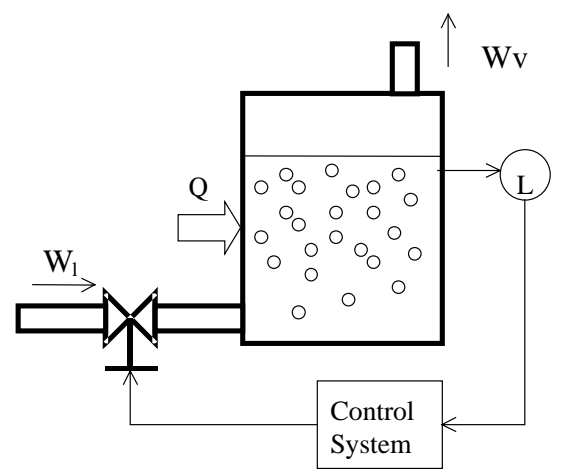

Figure 6. Schematic diagram of simplified steam generator model

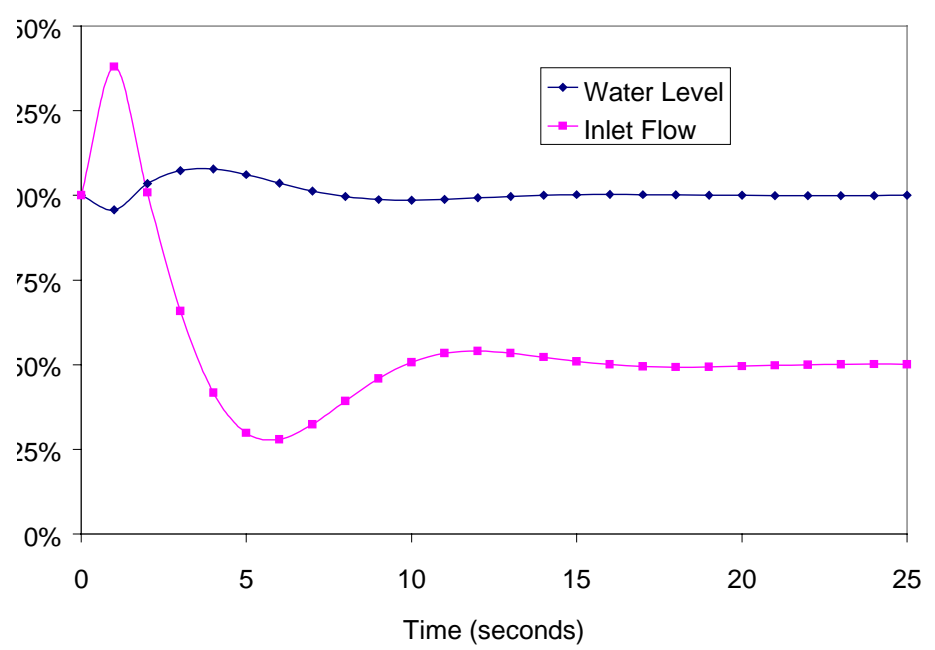

Figure 5. Control system responds to prevent scram following a power transient from $100 \%$ to $50 \%$ power

\section{Task 2. Advanced Monitoring and Diagnostics}

The objective of this research task is to develop an on-line monitoring system for fault detection and isolation (FDI) of sensors and field devices in a nuclear power plant. In this research emphasis has been given to process instrumentation in a nuclear power plant such as temperature, pressure, flow, level transmitters, and measurements of control functions. Field devices include valve actuators, control modules, spray and heater systems, pumps, and other similar equipment. The goal of this task is to provide diagnostics information to a system executive for enhanced decision-making by the plant control system.

The following R\&D tasks have been accomplished during Phase-I:

\section{Task 2.1 Data-Driven Prediction of Process Variables}

Under this task we have completed the development of data-driven models for the characterization of sub-system dynamics for predicting state variables, control functions, and expected control actions. We have also developed the Principal Component Analysis $(P C A)$ approach for mapping system measurements, and a nonlinear system modeling approach called the Group Method of Data Handling $(G M D H)$ with rational functions.

\section{Task 2.2 Detection of Simultaneous Faults}

Under this task, we have completed the development of a fault detection and isolation module that combines system operational knowledge (including system simulation) and a rule-based logic for both single and dual faults in dissimilar sensor and field devices. In addition, we have developed a complimentary approach that quantifies the prediction errors using a fault pattern classification technique.

The above techniques have been applied to a laboratory process control loop using both simulation and actual loop measurements. The techniques have been demonstrated for 
detecting and isolating faults in sensors and devices in a U-tube steam generator (UTSG) in a pressurized water reactor (PWR). Only simulation data were used in the latter case. Full details of this work are documented in Attachment IV. The application of the group method of data handling (GMDH) for state prediction and the expert system module is illustrated by application to a laboratory process control loop shown in Figure 7. A drift fault was imposed on the tank water level sensor. Using the previously established models, experimental data were used to predict tank inlet flow rate, outlet flow rate, controller output, and tank water level. The following rule set is used to conclude that the faulty device is the water level sensor.

IF (Outlet Flow rate Error $>$ Threshold)

AND (Water Level Error $>$ Threshold)

AND (Outlet MOV Position Error < Threshold)

THEN Loop Faulty Component $=$ Water Level Sensor

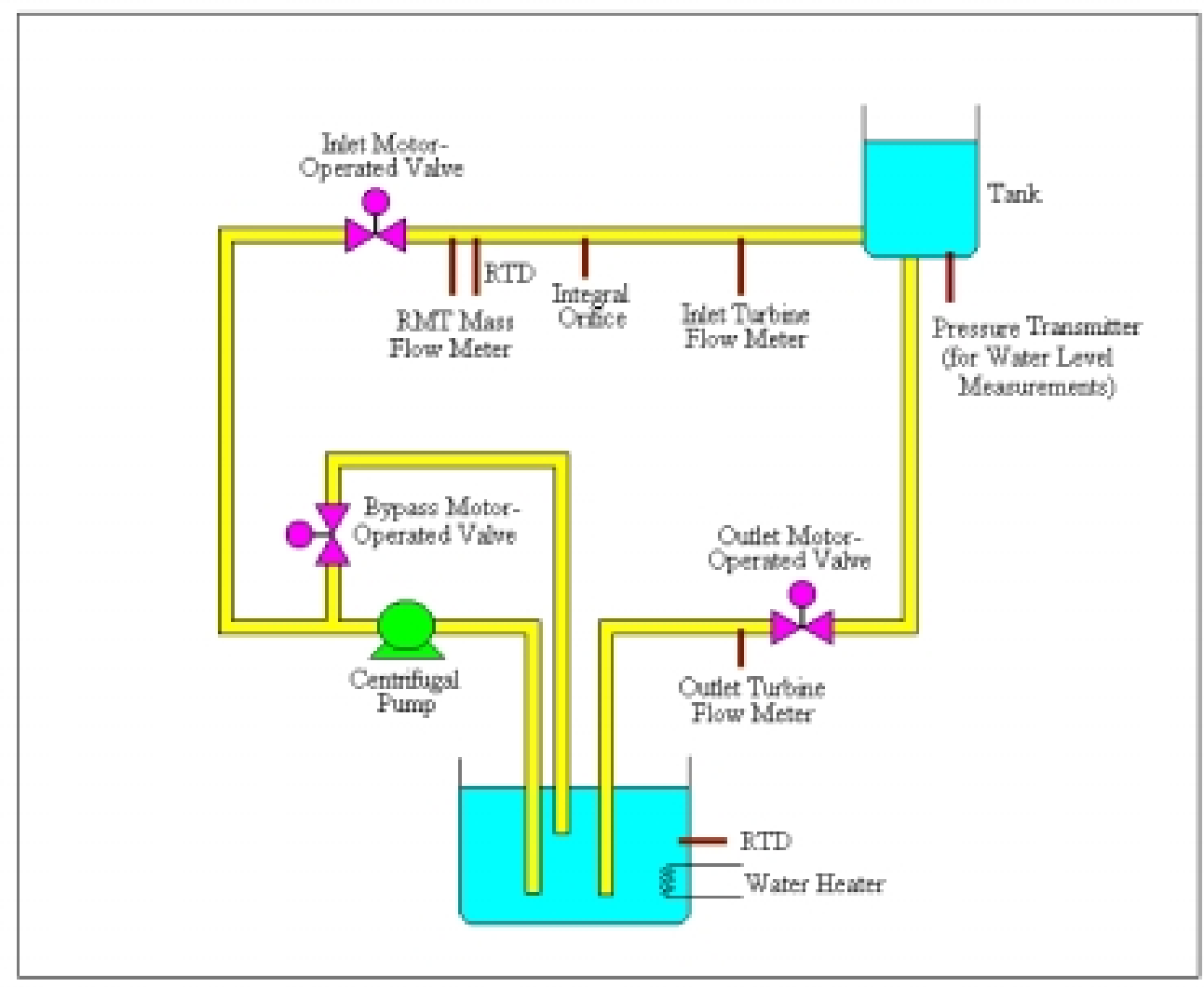

Figure 7. A schematic of the low-pressure water loop system, showing various sensors and field devices. 


\section{Task 3. Nuclear Power Simulation and Reliability Methods}

\section{Task 3.1 Plant Model Refinement}

Since 1996, a full plant engineering simulation code has been under development and in use at North Carolina State University for simulating the dynamic response of pressurized water reactors during normal operational transients as well as design basis events, with the exception of LOCA. The model is currently capable of simulating multi-loop PWR systems with U-Tube steam generators including asymmetric loop operation. Both primary and secondary sides are represented, including balance of plant and control systems. Plant protection systems are modeled including the normal spectrum of reactor trips and engineered safety features.

The work under this task required improvements in the plant simulator, which consist of refinements to existing models as well as development of new models to support the complete secondary side modeling effort. The secondary side has been broken into three separate subsystems. The first subsystem consists of the main steam system from the steam generators to the turbine and includes the main steam safety valves, steam dump system and the turbine bypass system. Figure 8 illustrates the current model of the main steam system. In addition, refinements to existing models have been oriented toward improved component or sensor models and include 1) development of a new valve model which allows for arbitrary dead band (hysterisis) and time constant, 2) a general level sensor $(\Delta \mathrm{P})$ model which allows for the prediction of both true and indicated water level and 3) a RTD model for the prediction of fluid temperatures. Options have been added to

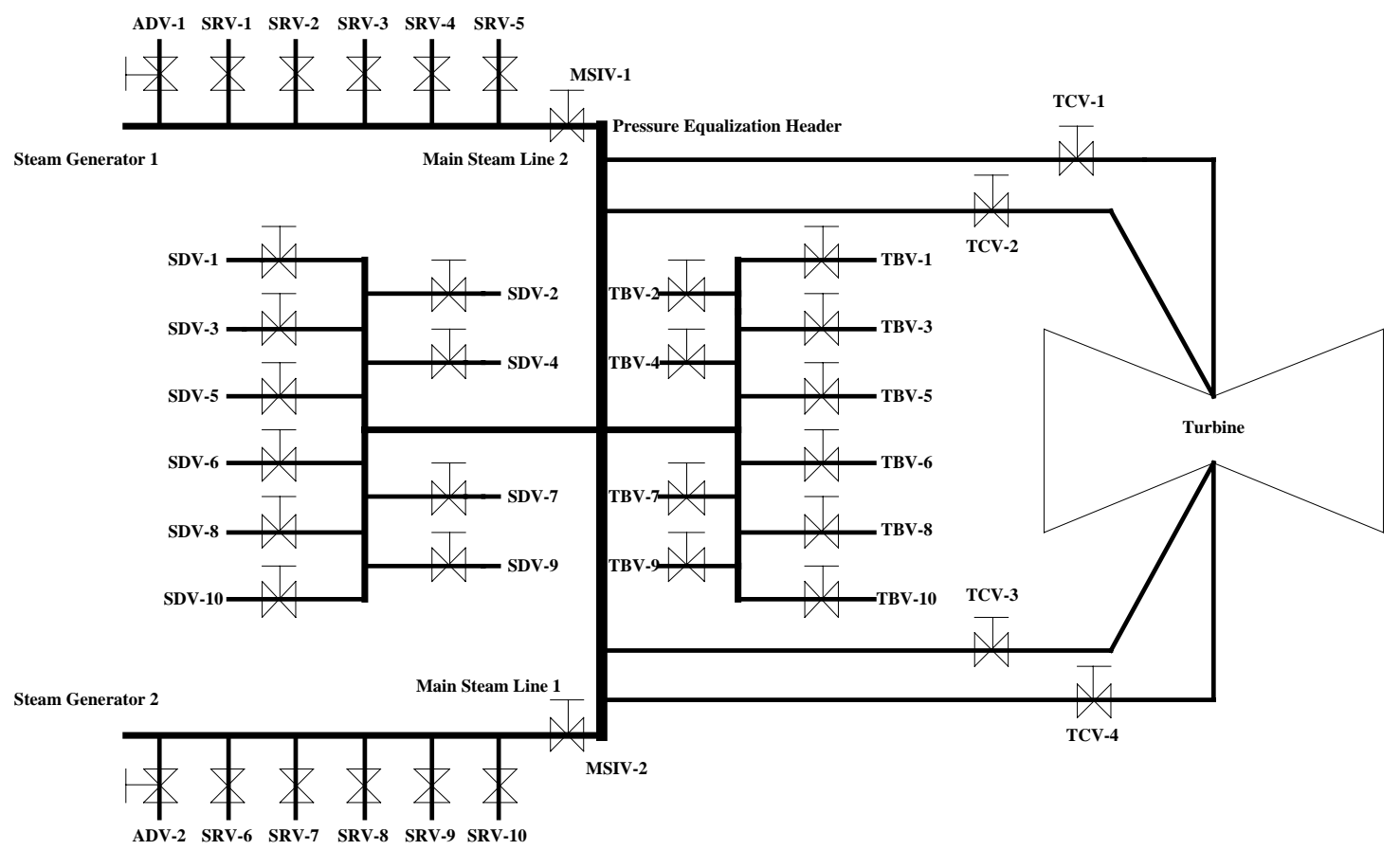

Figure 8. Main Steam System 
the code to allow for degradation in the heat transfer across the steam generator from both fouling and blocked or plugged tubes, as well as the corruption of sensor outputs through step and ramp changes in sensor output with arbitrary levels of random noise.

\section{Task 4. Nuclear Information System Architecture and Integration}

The objective of this task to promote the development of an integrated control and information system architecture for future nuclear power plants that will provide the processing support for self-organizing control applications while isolating the algorithms themselves from hardware-dependent interactions. The research approach we are employing is to develop the concept of a Plant-Control Computing Environment (PCCE), identify functional requirements for such a computing environment, and demonstrate the PCCE concept. The PCCE demonstration will be accomplished through the development of key architectural components for use in a prototype application involving integrated balance-of-plant control and diagnostics functions.

\section{Task 4.1 Requirements for Information-System Architecture}

Our Phase I research addressing the control and information system architecture for future nuclear power plants involves the evolution of the PCCE concept and the generation of functional requirements. For Phase I of this research we have generated functional requirements addressing

- general design attributes,

- human-system interface requirements,

- control application interface requirements,

- computing platform interface requirements,

- monitoring and control requirements,

- fault handling and recovery requirements,

- system management requirements, and

- configuration requirements.

Attachment VI provides the full description of the PCCE concept and documents the high-level functional requirements that express the capabilities envisioned for the control application environment. The functional requirements for the PCCE are the product of this activity. A prototype demonstration of the PCCE concept will be accomplished in subsequent phases of this project and the functional requirements will be revised based on the associated research findings.

\section{Task 5. Project Integration}

For Phase I, this is mostly an administrative task of integration between the different participants: ORNL, UTK, and NCSU. The final objective of this task is to integrate modeling and diagnostics within the final control engine demonstration. 


\section{Attachments}

The following attachments are included

Attachment I. Adaptive Inverse Control Algorithms

Attachment II. Control-Priority Mode Selection Algorithm

Attachment III. Requirements-Driven Control System Design

Attachment IV. Advanced Monitoring and Diagnostics

Attachment V. Nuclear Power Simulation and Reliability Methods

Attachment VI. Nuclear Information System Architecture and Integration 